\title{
La expedición americana de Alexander von Humboldt y su contribución a la ciencia del siglo XIX
}

L'expédition américaine d'Alexandre von Humboldt et sa contribution à la science du XIXo siècle

The American Expedition of Alexander von Humboldt and its Contribution to 19th-century Science

Sandra Rebok

\section{OpenEdition}

Journals

Edición electrónica

URL: http://journals.openedition.org/bifea/6080

DOI: $10.4000 /$ bifea.6080

ISSN: 2076-5827

\section{Editor}

Institut Français d'Études Andines

\section{Edición impresa}

Fecha de publicación: 1 diciembre 2003

Paginación: 441-458

ISSN: 0303-7495

Referencia electrónica

Sandra Rebok, "La expedición americana de Alexander von Humboldt y su contribución a la ciencia del siglo XIX », Bulletin de l'Institut français d'études andines [En línea], 32 (3) | 2003, Publicado el 08 diciembre 2003, consultado el 10 diciembre 2020. URL : http://journals.openedition.org/bifea/6080 DOI : https://doi.org/10.4000/bifea.6080

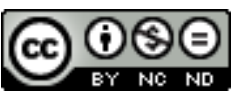

Les contenus du Bulletin de l'Institut français d'études andines sont mis à disposition selon les termes de la licence Creative Commons Attribution - Pas d'Utilisation Commerciale - Pas de Modification 4.0 International. 


\title{
LA EXPEDICIÓN AMERICANA DE ALEXANDER VON HUMBOLDT Y SU CONTRIBUCIÓN A LA CIENCIA DEL SIGLO XIX
}

\author{
Sandra REBOK
}

\begin{abstract}
Resumen
Este trabajo estudia el significado de las elaboraciones y publicaciones científicas de Alexander von Humboldt — que resultaron de su famosa expedición americana (1799-1804)— para el desarrollo de las ciencias de su época. Tradicionalmente en todo tipo de escritos se enfatiza la inmensa aportación de este viajero prusiano a la investigación científica. Aquí será analizada más detalladamente y críticamente en qué consiste exactamente dicha aportación. Se estudiará la repercusión que ha tenido tanto la gran información sobre América como su nuevo concepto interdisciplinario y holístico de la ciencia. No nos interesa clasificar su aportación según las distintas disciplinas académicas, sino en su visión general, ver lo novedoso y valioso de su información, su planteamiento científico así como su método para el avance de las ciencias tanto en Europa como en América.
\end{abstract}

Palabras claves: Alexander von Humboldt, viajeros, historia de las ciencias, expediciones.

\section{L'EXPÉDITION AMÉRICAINE D'ALEXANDRE VON HUMBOLDT ET SA CONTRIBUTION À LA SCIENCE DU XIX ${ }^{\circ}$ SIÈCLE}

\section{Résumé}

Cet ouvrage étudie le sens des élaborations et des publications scientifiques d'Alexandre von Humboldt — fruits de sa fameuse expédition américaine (1799-1804) — pour le développement des sciences de son temps. Dans tous les écrits on emphatise généralement l'immense contribution de ce voyageur prussien à la recherche scientifique. Nous analyserons ici plus en détails et de façon critique la nature exacte de cet apport. On étudiera les répercussions de la somme d'informations sur l'Amérique comme son concept interdisciplinaire novateur et holistique de la science. La classification selon les différentes disciplines académiques ne nous intéresse pas et lui préférerons l'étude, dans sa vision générale, du côté novateur et précieux de son information, son approche scientifique et sa méthode pour le progrès des sciences en Europe et en Amérique.

Mots clés Alexandre von Humboldt, voyageurs, histoire des sciences, expéditions.

\footnotetext{
* Instituto de Historia de la Ciencia . Consejo Superior de Investigaciones Científicas, Madrid. E-mail: srebok@ceh.csic.es
} 


\title{
THE AMERICAN EXPEDITION OF ALEXANDER VON HUMBOLDT AND ITS CONTRIBUTION TO 19TH-CENTURY SCIENCE
}

\begin{abstract}
The aim of this study is the significance of the development of the Alexander von Humboldtpublications-, resulting from his famous American expedition (1799-1804). Various authors have stressed the immense contribution of this Prussian voyager to scientific investigation. Here I analyze critically and in greater detail the precise nature of his contribution examining the impact of both the great information about America, and his new interdisciplinary and holistic concept of science. The goal of this work is not to classify his contribution to distinct academic disciplines. I attempt to study the impact of his greater vision and to see what was new and valuable in his accounts and his scientific investigation, as well as his method for the advance of science in both Europe and America.
\end{abstract}

Key words: Alexander von Humboldt, Voyagers, History of Science, Expeditions.

"La importancia de Humboldt para la ciencia, tanto por sus propios trabajos como por el impulso que comunicó, (...), es superior a todo encomio: así obró por sus descubrimientos como por sus escritos científicos, como por su ejemplo y su protección; el mismo aliento que infundió con la palabra escrita, lo fomentó con todos los recursos que le prestaban su posición, su riqueza y su celebridad universal. Su influencia en todos los sentidos es imposible avalorarla. Él fué uno de aquellos que con poderosa mano empujaron las ciencias naturales por la senda del progreso que vienen recorriendo desde fines del pasado siglo. En todas las ramas de esta vasta serie de conocimientos, productos casi todos de la época moderna, que se comprenden bajo el nombre de ciencias naturales, ha trabajado él, ha descubierto nuevos hechos, ha revelado nuevas verdades: en todas las esferas del humano saber donde sentó la planta, ha dejado profunda e indeleble huella de su paso" (Font y Guitard, 1862: 130).

Esta cita, tomada de una necrología sobre Alexander von Humboldt, que retomaremos más adelante, nos sirve para demostrar el énfasis que tradicionalmente se ha puesto en demostrar la importancia que Alexander von Humboldt tuvo en el desarrollo de las ciencias.

A primera vista, destacan varios aspectos de su famosa expedición americana (1799-1804), que contribuyeron, en términos generales, al éxito y difusión que alcanzó y, de forma particular, a la evolución de las ciencias en esta época. Lo innovador de la expedición fue su carácter privado y su independencia económica, lo cual le dio al sabio alemán una libertad absoluta en sus decisiones sobre la ruta, los objetivos y los contenidos de sus investigaciones. Por otra parte, hay que destacar el carácter innovador del proyecto científico de Humboldt — especialmente su concepto de una investigación holística- que llevó a cabo tanto durante el viaje, como en la etapa posterior de estudio y elaboración de los resultados. Además, en este viaje exploró regiones de América que no habían sido exploradas anteriormente, y utilizó los más avanzados instrumentos 
de medición que había conseguido en París. De esta manera fue capaz de obtener nuevos datos sobre el continente americano y, en consecuencia, pudo contribuir en una medida considerable al avance del conocimiento del Nuevo Mundo.

En este trabajo nos proponemos analizar de forma detallada y crítica en qué consiste esta aportación, a qué aspectos se refieren los elogios que sobre Humboldt y su obra se repiten en muchos textos, como en el ejemplo citado, y cuáles han sido los descubrimientos y observaciones más novedosas o valiosas de la obra del viajero prusiano. En nuestro estudio revisaremos el significado que tuvieron los escritos de Humboldt para las ciencias de aquel tiempo, ya que hay que diferenciar claramente entre la relevancia que adquirió en el siglo XIX (incluso finales del XVIII) y la validez o repercusión de sus logros científicos hoy día. De manera específica, nos interesa demostrar el impacto que tuvieron sus contribuciones científicas en publicaciones a muy corto plazo, para comprender y valorar la visión europea de la obra de Humboldt, durante su vida y en los años inmediatos a su muerte.

Con este objetivo, resulta obvio la necesidad de recurrir a las publicaciones de entonces para poder contrastar esta visión con la que se tiene en la actualidad. Para ello, se han elegido tres fuentes principales: la ya mencionada necrología, escrita por Juan Font y Guitard y publicada en el año 1862 en la revista científica barcelonesa $L a$ Abeja; la biografía científica sobre Humboldt editada en 1872 por Karl Bruhns, es decir pocos años después de su muerte en 1859; y, para revelar la visión del propio Humboldt sobre estas cuestiones, se utiliza principalmente su famosa obra, el Cosmos (1874-75), un trabajo sintético elaborado a finales de su vida, así como alguna correspondencia. De este modo - y de manera representativa - se logra mostrar una visión científica, la percepción pública difundida en la prensa y la versión personal de nuestro famoso viajero.

En este contexto será interesante ver si la valoración de Humboldt sobre aquellos aspectos de su trabajo que consideró la esencia de su labor científica, coincide con la percepción exterior. A fin de analizar y presentar dichos aspectos, se estudiará de manera diferenciada lo que podemos considerar sus aportes a las distintas ramas de la ciencia, de lo que se valora como su contribución general.

\section{VISIÓN FRAGMENTADA: APORTES ESPECÍFICOS A LAS DISTINTAS RAMAS DE LA CIENCIA}

Examinando el conjunto de los trabajos científicos realizados por Humboldt,

"uno advierte que se trata de asuntos relacionados con disciplinas muy diversas, que van de la astronomía a las ciencias de la atmósfera; de la superficie de la tierra (la geografía, la orografía y la hidrología) hasta el centro de la misma (la geología y la vulcanología); de la estructura social (o sea, la economía, el comercio, la demografía y la estadística) hasta la historia del país, en suma las ciencias que le interesan a Humboldt van del centro de la tierra a la bóveda celeste: pasan por el hombre y la sociedad" (Labastida, 1999: 45).

Aunque en esta cita falten algunos aspectos de la obra de Humboldt, describe bien el conjunto de sus actividades científicas, y demuestra además, cómo ya en las 
distintas disciplinas elegidas se manifiesta su interés por el todo. Es evidente que existe una diferencia tanto en el significado como en la validez de su aportación en estas distintas áreas del saber; mientras en algunas realizó un trabajo pionero, en otras solamente aportó datos y observaciones nuevas, que se podían añadir o contrastar con los datos ya conocidos.

Es conocido que, ya antes de su viaje americano, Humboldt había realizado importantes estudios científicos como por ejemplo, sus investigaciones biológicas sobre la distribución de plantas y sus condiciones ecológicas, los experimentos químicos y electrobiológicos con organismos vivos (1803), en especial sus estudios galvánicos, así como trabajos sobre la forma y la superficie de la tierra relacionados con datos climáticos y físicos (Jahn, 2001). La amplia formación del sabio prusiano le posibilitó adentrarse en otras disciplinas como la física biológica, la química, o la medicina. Sus logros en el campo de la medicina teórica y la medicina general forman parte de los principales avances en la fisiología moderna (Schipperges, 1990: 64), aunque sus aportes no han sido tan reconocidos como en otras ciencias, debido a su carácter fragmentario y, en algunos casos, a la debilidad de sus argumentaciones entre sus propios coetáneos.

Fue este conocimiento universal y puntual sobre los últimos logros científicos lo que le sirvió a Humboldt para suministrar a toda la comunidad académica y al público en general, del modo en que lo hizo, la valiosa e innovadora información que había recogido sobre el continente americano, cargadas con anotaciones, observaciones, mediciones y análisis que él mismo realizó, conforme con su esquema científico. La transmisión que de su conocimiento se llevó a cabo no fue global, sino que fue fraccionada y dirigida a distintas especialidades que correspondían a la nueva clasificación que se hizo de la ciencia durante el siglo XIX, y sobre todo en la segunda parte.

Un buen ejemplo de la división de su obra según las disciplinas es la biografía editada por Karl Bruhns en 1872, que demuestra muy bien la percepción científica de los logros de Alexander von Humboldt que se tenía pocos años después de su muerte. Mientras los primeros dos tomos se dedican a describir cronológicamente la vida de Humboldt, el tercero analiza su quehacer científico, clasificándolo en las distintas áreas.

Las investigaciones aisladas de distintos autores, más o menos coetáneos, sobre las contribuciones de Humboldt en diferentes materias, nos sirve para saber cuáles de sus logros fueron considerados más relevantes. Una enumeración larga de la que podemos destacar aspectos relacionados con las matemáticas, astronomía y geografía matemática, algunos estudios físicos y químicos sobre el magnetismo de la tierra, la meteorología, geología, geografía, antropología, historia, geografía de las plantas y botánica, zoología, anatomía comparada y finalmente la fisiología. Sin embargo, es significativo que no haya ningún capítulo sobre lo que él mismo consideraba que era más relevante en su obra: el desarrollo de un concepto holístico y el concepto global de la geografía física.

En esta evaluación histórica del trabajo de Humboldt, los autores destacaron la parte empírica. Así consta al astrónomo Karl Bruhns, quien piensa que la orientación de Humboldt estaba menos dirigida a crear algo en las ciencias exactas, que a recoger colecciones (Bruhns, 1969, t. 3: 3). También el meteorólogo Alfred Dove se muestra 
crítico, diciendo que los propios progresos de las ciencias que se deben a Humboldt, se dejan contar y medir fácilmente (Bruhns, 1969, t. 3: 481). Creemos que la evaluación posterior del significado de las actividades científicas de Humboldt estaba marcada por las controversias sobre el mismo carácter y la función de las ciencias, es decir sobre el papel de experiencia y reconocimiento, observación y teoría, ilustración y "reacción” romántica (Dettelbach, 2001: 138).

Esta manera fraccionada de interpretar a Humboldt contribuyó a que su obra fuera menos valorada en las siguientes décadas, ya que muchos de sus resultados específicos así como las mediciones no alcanzaron el nivel científico de la época, siendo superados en los siguientes años debido a una considerable mejora en la construcción, y consecuencia, en la precisión de los instrumentos de medición.

Ante esta visión crítica hay que tener en cuenta que Humboldt no practicó un mero trabajo de análisis, ni que tan sólo descompuso el cuerpo complejo en sus simples partes, sino que sintetizó sus elaboraciones. Esta labor de síntesis la pudo realizar gracias al concepto interdisciplinario que tenía de la ciencia. De hecho, el mérito de Humboldt no se debió tanto a logros específicos en un determinado campo de la ciencia, sino a su capacidad de detectar y analizar las conexiones entre los fenómenos. Su significado está en su concepto científico holístico, su manera de crear lazos entre las distintas disciplinas para comprender y representar el Nuevo Mundo en sus diversos aspectos a través de las colecciones traídas a Europa, sus descripciones — también representadas en dibujos de carácter científico-, y sus reflexiones teóricas.

La obra de Alexander von Humboldt tiene que ser valorada —igual que su programa científico - de una manera holística, es el TODO lo que ha tenido su gran impacto $y$, en menor medida, las distintas partes que la componen.

\section{VISIÓN GENERAL: APORTES A LA CIENCIA GLOBAL}

Este concepto holístico, elaborado por Humboldt en las últimas dos décadas de su vida, ya estaba presente en sus años jóvenes - como demuestra su correspondencia de aquellos años (Jahn \& Lange, 1973)—. Desde el principio de su actividad científica, Humboldt formuló lo que él consideraba el fin de sus investigaciones: comprender y describir el Nuevo Mundo desarrollando la idea holística de una geografía física (se pueden originar confusiones por utilizar el mismo término para fenómenos distintos, o a la inversa, llamar el mismo fenómeno de diferentes maneras. El propio Humboldt, al definir el objetivo de sus investigaciones, utilizó los términos física del mundo, teoría de la tierra y geografía física como sinónimos). Ya en el año 1793 se le atribuía como gran mérito científico el haber definido y aclarado metodológicamente lo que él llamaba physique du monde (Beck, 1978: VIII); entre 1795 y 1799 Humboldt planeó una descripción general de una geografía mundial de plantas y de las interrelaciones en el ciclo vital con las fuerzas físicas de la tierra (Jahn, 2001: 32).

Su memoria sobre su "vida literaria", redactada en 1799 para Carlos IV, donde define el objetivo de su viaje, demuestra que esta idea ya estaba presente antes de su salida a América: 
“(...) de voir une autre partie du monde y de la voir sous les rapports de physique générale, d'étudier non seulement les espèces et de leur caractères, étude au quel on s'est voué trop exclusivement jusqu'ici, mais l'influence de l'Atmosphère et de sa composition chymique sur les corps organisés; la construction du globe, l'identité des couches dans les pays les plus éloignés les uns des autres, enfin les grandes harmonies de la Nature (...)" (Puig-Samper, 1999: 354).

Asimismo, este escrito revela el interés de Humboldt de buscar la correlación entre los diferentes componentes de su física del globo, y de estudiar de qué manera estos componentes eran influidos tanto por otros elementos como por el medio ambiente en que se encontraban. Este método lo aplicó en todas sus investigaciones, tanto para estudiar las relaciones entre plantas y minerales, clima y vegetación como entre el hombre y su entorno. En sus obras estudia y discute los problemas que ve entrelazados entre todo tipo de fenómenos, es decir entre la geografía de las plantas y la geología, entre la historia de la política y la moral del ser humano, entre la naturaleza y el espíritu del hombre. Con estas conexiones surgieron cuestiones científicas fundamentales relacionadas tanto con la historia de la tierra como con la historia humana; preguntas que Humboldt se planteó resolver con sus investigaciones.

En la introducción de su obra Relation historique explica lo que asociaba con su idea de una física del mundo:

"Un doble fin me había propuesto en el viaje cuya relación histórica publico ahora. Deseaba que se conociesen los países que he visitado, y recoger hechos que diesen luz sobre una ciencia apenas bosquejada y asaz vagamente designada con los nombres de Física del mundo, de Teoría de la tierra, o de Geografía Física. De ambos objetos parecióme más importante el segundo. (...) prefiriendo siempre al conocimiento de los hechos aislados, aunque nuevos, el del encadenamiento de los hechos observados largo tiempo ya, parecíame mucho menos interesante el descubrimiento de un género desconocido, que una observación sobre las relaciones geográficas de los vegetales, sobre la migración de las plantas sociales, sobre el límite de altitud a que se elevan sus diferentes tribus hacia la cima de las Cordilleras.”( Humboldt, 1991a, v. I: 4)

Antes de empezar su gran obra sintetizadora, el Cosmos, Humboldt expresa de manera muy explícita, en una carta que manda el 24 de octubre de 1834 a Varnhagen von Ense, su idea de comprobar la unidad de la naturaleza en sus diferentes partes y de describir los rasgos básicos en los que consiste todo el universo en una sola obra:

"Yo tengo la espléndida idea de presentar todo el mundo material, todo lo que sabemos hoy de las aparencias del espacio y de la vida en tierra, desde las estrellas nebulosas hasta la geografía de los musgos en las rocas de granito, en una sola obra, y en una obra que inspira con un idioma vivo y deleita el alma. Cada idea grande e importante, que

(1)“Ich habe den tollen Einfall, die ganze materielle Welt, alles was wir heute von den Erscheinungen der Himmelsräume und des Erdenlebens, von den Nebelsternen bis zur Geographie der Moose auf den Granitfelsen, wissen, alles in einem Werke darzustellen, und in einem Werke, das zugleich in lebendiger Sprache anregt und das Gemüht ergötzt. Jede grosse und wichtige Idee, die irgendwo aufglimmt, muss neben den Thatsachen hier verzeichnet sein". 
surge en cualquier sitio, tiene que estar mencionada al lado de los hechos." (Humboldt, 1860: 20) (1)

Al examinar su obra bajo este criterio, vemos que todo su programa de investigación, todo su pensamiento holístico está basado en esta idea de una geografía física, lo que hacia el final de su vida extendió a una descripción física del mundo, con la unificación de todo lo creado en la tierra con el espacio. En su prólogo al Cosmos explica más detalladamente lo que pretende con su investigación, sobre todo lo relacionado con su concepto holístico. Este hecho demuestra que Humboldt no creía que el valor especial de su obra fuera para las ciencias singulares, sino que consideró sus estudios como trabajos preliminares, en cierto sentido, como camino que llevaba a su objetivo:

"Las vicisitudes de mi vida y el ardiente deseo de instruirme en muy diferentes materias, me obligaron a ocuparme durante muchos años, y exclusivamente en aparencia, en el estudio de ciencias especiales, como la botánica, la geología, la química, la astronomía y el magnetismo terrestre. Preparación necesaria era ésta, si habían de emprenderse con utilidad lejanos viajes; pero también tales trabajos tenían otro objeto más elevado: el de comprender el mundo de los fenómenos y de las formas físicas en su conexión y mutua influencia. Desde mi primera edad he tenido la suerte de escuchar los benévolos consejos de hombres superiores, convenciéndome desde luego de que si no se poseen sólidos conocimientos relativamente a las diversas partes de las ciencias naturales, la contemplación de la Naturaleza en más extensos horizontes, como el intento de comprender las leyes porque se rige la física del mundo, solo vana y quimérica empresa serían. Los conocimientos especiales se asimilan y fecundan mutuamente por el mismo enlace de las cosas. (...) De este modo es como el naturalista ávido de saber se ve conducido de una esfera de fenómenos dada a otra segunda que limita los efectos de aquella." (Humboldt, 1874-75, v. I: VII-VIII)

En la continuación de esta cita, Humboldt ya señala una de sus principales aportaciones personales:

"La mayor parte de los viajeros que han verificado expediciones científicas, se limitaron a visitar costas, y así necesariamente tiene que suceder en los viajes alrededor del mundo; yo he disfrutado de la ventaja de haber recorrido espacios considerables en el interior de dos grandes continentes, y en regiones en que presentan los más fuertes contrastes (...).”

Para lo que nos proponemos, consideramos el Cosmos como una fuente muy válida, ya que refleja la visión que Humboldt tenía de su obra final, al término de su vida. Es interesante ver qué aspectos de su obra él resalta como los más importantes para la evolución de las ciencias. Hemos visto que Humboldt menciona en este contexto su exploración científica de regiones poco exploradas o completamente desconocidas, pero sobre todo su concepto globalizador de las ciencias, en las que — ampliando su término- no solamente se reúne el conocimiento que ha logrado obtener sobre la tierra, sino sobre el mundo entero. Esto queda reflejado también en el título que elige para su obra culminante al final de su vida: Cosmos. Ensayo de una descripción física del mundo. 
Sin embargo, hay que ser consciente de que el desarrollo de esta visión del mundo era fruto de su estudio de las corrientes filosóficas de su tiempo. Es decir, Humboldt no inventó este concepto holístico — como veremos más adelante, él mismo hace referencia a los primeros cronistas españoles como precursores - sino que lo sintetizó, lo elaboró y además de utilizarlo para sus reflexiones teóricas, lo llevó a cabo en su propia investigación (Humboldt, 1874-1875, v. I: VII-VIII).

Si bien el germen de estos conceptos los había encontrado en antiguos escritos, su metodología se deriva de la síntesis de las corrientes de pensamiento presentes en la época;

"Es aquí donde confluye el Racionalismo, como eje de su formación ilustrada; el Empirismo, como instrumento metodológico, y el Romanticismo, como ruptura que representa con el concepto de unas ciencias de la Naturaleza que consideran el mundo formado por fenómenos compartimentados y clasificables.” (Monreal Sanz \& Álvarez Falcón, 2001, v. I: 354).

Sin duda el filósofo de mayor influencia sobre Humboldt fue Emmanuel Kant, especialmente en lo que concierne a sus ensayos "Geografía Física" y "Historia general de la naturaleza del firmamento".

Otra aportación significativa de Humboldt a las ciencias fue ser el precursor en la popularización de las ciencias, ya en la primera mitad del siglo XIX. En el fenómeno de la popularización se distinguen tres etapas (Daum, 1998: 269): la publicación de Tableaux de la nature, por primera vez en 1808 — con su primera edición castellana en 1876-, las famosas conferencias sobre su idea del cosmos que impartió en Berlín durante el invierno 1827-1828, y, finalmente, la publicación de su opus magnum, el Cosmos, editado en su versión original en alemán entre 1845 y 1862, cuya traducción al español vio la luz a partir de 1851.

Sobre todo en sus lecturas se manifiesta su intención no solamente de percibir y explicar el cosmos con las interconexiones entre sus partes como un todo, sino de presentar los conocimientos sobre las ciencias naturales a un público amplio. Humboldt no buscó tener como espectadores solamente a la elite científica de entonces, sino que quiso llevar el conocimiento al gran público. Con este fin, impartió 61 lecturas sobre lo que él percibía como geografía física en la Universidad de Berlín, y, debido al gran éxito obtenido, dictó una serie comprimida de 16 conferencias en la Singakademie de Berlín.

Esta búsqueda de divulgar el conocimiento, de ponerlo en palabras comprendidas por los no letrados, fue recogida en una de sus necrologías que reproducimos a continuación:

"Por él vinieron a ser las ciencias naturales lo que son hoy día, no, cual antes, patrimonio exclusivo de los eruditos, no meras servidoras de la medicina y de las artes, sino el más poderoso medio de cultivo de la humana inteligencia, la gran palanca de ilustración del pueblo. No existieron antes escritos populares; (...) antes merecían ser llamados impopulares por la árida que presentaban a la ciencia, escarpada e inaccesible.” (Daum, 1998: 131) 
Sin duda, las obras de Humboldt también han influido ampliamente en la concepción del mundo americano y de esta manera han contribuido a modificar o redefinir la imagen que se tenía del Nuevo Mundo en la Europa del siglo XIX, muy distinta de la que se tenía en el siglo XVIII.

Desde su regreso de América, Humboldt comenzó a ocuparse de manera intensa en el estudio de la historia de las colonias españolas, ya que pensaba que hay que conocer el pasado de una región para comprender su presente. Para ello, utilizó, sobre todo, las antiguas fuentes españolas (Minguet, 1985, t. II: 401-404), es decir, los relatos de los primeros cronistas, misioneros, viajeros y funcionarios, así como las recopilaciones e interpretaciones de aquellos escritos a lo que se dedicaron los investigadores en los siglos XVII y XVIII (Rebok, 2001). Como Humboldt dice:

“(...) la lectura de las obras que contienen las narraciones de los conquistadores ha tenido para mí especial atractivo, y las investigaciones hechas en algunos archivos de América y en bibliotecas de diferentes partes de Europa me han facilitado el estudio de una rama descuidada de la literatura española" (Humboldt, 1992: 12).

Una referencia muy interesante en este contexto la encontramos en un capítulo dedicado al "Desarrollo de la idea del Cosmos en los siglos XV y XVI" que se halla en el segundo tomo de su obra Cosmos. En él, Humboldt insiste en que los cronistas eran los precedentes de lo que él llama física del globo, ya que es la física la ciencia que contempla todos los aspectos a la vez:

"Cuando se estudian seriamente las obras originales de los primeros historiadores de la Conquista, sorprendenos encontrar en los escritores españoles del siglo XVI el germen de tantas verdades importantes en el orden físico. (...) la curiosidad impaciente de los primeros viajeros y de los que recogían sus narraciones, originó desde luego la mayor parte de las graves cuestiones que aún en nuestros días nos preocupan. (...) El fundamento de lo que hoy se llama física del globo, presciendiendo de las consideraciones matemáticas, se halla contenido en la obra del jesuita José Acosta, titulada Historia natural y moral de las Indias, así como en la de Gonzalo Hernández de Oviedo, que apareció veinte años después de la muerte de Colón. En ninguna otra época, desde la fundación de las sociedades, se ha ensanchado tan repentina y maravillosamente el círculo de las ideas, en lo que se refiere al mundo exterior y a las relaciones del espacio (...)" (Humboldt, 1874-1875, v. II: 255 y ss.).

Investigando y estudiando casi todas las obras españolas relacionadas con este tema, desde el descubrimiento de América hasta su tiempo, Humboldt llegó a ser un conocedor excelente de la historiografía española clásica, lo que le permitió valorar en su justa medida estas obras, que, por otra parte, en ese momento, tenían una escasa consideración en el ambiente científico; al ser catalogadas como exageradas, se mantenía un cierto escepticismo hacia ellas, y en Europa se las despreciaba como "cuentos de frailes" (Minguet, 1985, t. II: 12). Sacando estos cronistas españoles del olvido y utilizando sus escritos como material científico, hizo que se recuperaran y se pusieran estas valiosas fuentes a disposición de la ciencia, lo cual constituye, de forma indirecta, una contribución a esta. 
El estudio profundo de las fuentes hispanas y del pasado colonial español se refleja en sus obras. A la vista están las numerosas referencias que hace a los escritos de los cronistas, sobre todo en tres de sus obras Sitios de las Cordilleras y monumentos de los pueblos indígenas (Humboldt, 1878), Cristóbal Colón y el descubrimiento de América (Humboldt, 1992) y el Cosmos. Pero no solamente encontramos en ellas referencias a los cronistas. Por el tipo de comentario que hace Humboldt detectamos vínculos más profundos y, sobre todo, que el planteamiento global que presenta está relacionado con las ideas que contienen estos relatos sobre el Nuevo Mundo y su forma de percibirlo.

Su preocupación por el desarrollo de su concepto científico basado en el estudio de cronistas también fue un aspecto destacado en su necrología:

“(...) las más vastas consideraciones acerca del progresivo desenvolvimiento de la idea del Cosmos a través de todos los siglos, comprendiendo en ellas el influjo mutuo de los pueblos por las emigraciones, las conquistas, las invasiones; el predominio de las lenguas, la navegación y el comercio; los viajes y descubrimientos de continentes nuevos (...)" (Font y Guitard, 1862: 132).

Relacionado con lo dicho anteriormente, el naturalista alemán ofrece una visión optimista y defensora de América. En sus escritos contrapuso a la imagen de América creada por Raynal, Robertson, Buffon etc., una nueva percepción, en la que ofrece argumentos en contra de las visiones negativas. Humboldt formaría parte de la "disputa del Nuevo Mundo" al criticar y refutar los reiterados prejuicios eurocéntricos tanto en el campo natural como moral. Al pintar una imagen tan positiva de las colonias españolas, describiendo las maravillas de la naturaleza, en todos los sentidos, estudiando aquella realidad tan intensamente, Humboldt indirectamente argumentaba en contra de la idea de la supuesta inferioridad de América tan vigente en aquella época (Gerbi, 1982). Esto conduce a afirmar que Humboldt no se alineaba de ninguna manera dentro de esa leyenda negra, aquel mito acerca de las actitudes de los españoles en su forma de proceder en sus colonias, tan corriente dentro de la imagen de España en su tiempo.

La nueva imagen del continente también llega de forma gráfica. Antes de Humboldt, debido a la escasez de trabajos realistas para representar el Nuevo Mundo los artistas europeos recurrían muchas veces al material iconográfico de la Antigüedad y de la Edad Media en la que América se muestra como un mundo fantástico, habitado por monstruos y seres extraños, surgidos de las mitologías europeas y nutridos por las leyendas. Los viajeros que habían visitado estas lejanas regiones —con algunas excepciones - por lo general tenían pocas oportunidades para representar lo visto y manifestaban desinterés o una falta de capacidad artística para realizar tal labor. Por ello, Humboldt constituye uno de los iniciadores de esta renovación iconográfica en la representación de América, utilizando el dibujo para la representación científica, es decir, el trabajo artístico como complemento importante del trabajo científico. Esta contribución de Humboldt no solamente se realizó a través de sus propios dibujos, sino también gracias a la promoción de jóvenes artistas, a los que estimuló para que desarrollaran sus ideas de la pintura científica en América y plasmaran las formas de la naturaleza americana según los principios científicos y topográficos que él había fijado. 
Humboldt promovió lo que se puede llamar un "arte científico": la representación artística en el servicio de la ciencia. Esta representación iconográfica de la botánica, la zoología y sobre todo de los restos materiales de las culturas prehispánicas así como su expresión artística, como por ejemplo los monumentos prehispánicos, proporcionó una nueva imagen del mundo americano que hasta entonces estaba ampliamente dominada, más por la fantasía europea que por la realidad (Rojas-Mix, 1969). De hecho, a Humboldt se le atribuye un especial significado por la representación de los restos de los monumentos de los pueblos americanos. No era solamente el pionero al enfatizar la importancia de estos monumentos y su significado cultural, sino también el primero que aportó al arte europeo una representación científica de algunos de los monumentos más importantes (Rojas-Mix, 1969: 120).

Para llevar a cabo su obra recurrió a la arqueología y la paleontología, la antropología física, la geología así como a la lingüística e intentó recoger todo tipo de material sobre estas culturas precolombinas, bien restos arqueológicos, hallazgos paleontológicos, o leyendas y tradiciones generales. Los datos lingüísticos que había recogido los remitió a su hermano Wilhelm, quien los estudió y puso a disposición de la comunidad académica, logrando de esta manera ampliar el conocimiento que se tenía sobre las lenguas indígenas.

Por otra parte, Alejandro von Humboldt ayudó a cambiar y enriquecer la visión que se tenía de América. Hasta ese momento el continente sólo había sido considerado como el espacio donde se recogían datos; sin embargo, el sitio adecuado para la elaboración científica, o para escribir la historia de los viajes, expediciones y hallazgos parecía ser solamente Europa. Sus grandes centros como París, Londres o Madrid, se convertirían con sus museos o archivos en centros del saber europeo sobre el mundo no europeo, donde a los viajeros se les ofrecían las mejores condiciones para una tal elaboración científica de una expedición, o con otras palabras, de convertir una "aventura" en saber controlado y controlable, además de insertarla en un conocimiento preexistente y abrirla para la posterioridad.

Sin embargo, para Humboldt América también era el laboratorio para la experimentación y elaboración científica, más todavía, lo toma en consideración en su función como lector de sus escritos:

"Con el viaje de Humboldt se crea un espacio diferente para la escritura europea: el continente americano se convertirá ya no solamente en el teatro de las experiencias y hazañas de los europeos sino también en el lugar donde estas 'aventuras' (científicas) y sus resultados se fijarán para ser integrados al saber europeo sobre el otro.” (Ette, 2000: 54)

Otro aspecto que tampoco se debe dejar fuera de este análisis es su función como promotor de una colaboración científica que posibilitaría la creación de una red de conocimientos. En este sentido, Humboldt puede ser considerado como antecedente de la globalización del conocimiento científico al que nos vemos confrontados hoy día. Durante toda su vida estuvo preocupado no solamente en acumular datos, sino de intercambiarlos permanentemente con otros científicos en todo el mundo, a fin de divulgarlos y de contrastarlos con los resultados de otros colegas: 
"Humboldt había venido a ser el núcleo de la investigación científica, el centro adonde convergía la actividad de los naturalistas de todos los países. Las primeras noticias de los descubrimientos geográficos le eran dirigidas; numerosos observatorios le daban continua razón de los resultados de la contemplación astronómica; los nuevos y diarios portentos de la química, los adelantos de la física, las conquistas de las demás ciencias naturales se ponían desde luego en su conocimiento, y todos le enviaban sus libros, memorias y manuscritos." (Font y Guitard, 1862: 136)

Pero no promocionó solamente las ciencias, sino también personalmente a muchos científicos. A la vista está su influencia, su inspiración y su ayuda material a muchos viajeros que seguían sus huellas en las siguientes décadas y que continuaron ampliando y profundizando los conocimientos sobre el continente americano. Desde su vuelta a Europa hasta el final de su vida inspiró y apoyó a jóvenes científicos, viajeros o pintores en su proyecto de realizar una expedición. Con su ayuda -desde orientación ideológica, consejos concretos e informaciones científicas, envio de cartas de recomendación- muchos científicos accedieron de forma directa y más fácil a sus trabajos, instituciones y diferentes lugares, al igual que Humboldt pudo disfrutar en América de ciertas comodidades gracias a las cartas que llevaba de la Corte española. Su ayuda a veces rebasó lo meramente científico, ya no sólo era poner a su disposición de los más jóvenes los instrumentos científicos, sino que incluso les apoyó económicamente con parte de lo que le quedaba de su fortuna. Su figura, su saber y su disposición de enseñar y poner sus conocimientos al servicio de la sociedad inauguraba una nueva época en la que se incentivaba la investigación científica por todo el mundo, y particularmente en América. Además, hay que ser consciente de que no sólo hubo lo que se puede denominar una influencia directa — sobre todo por contacto personalsino también una influencia o inspiración indirecta sin que hubiera una relación personal. Varias de las expediciones realizadas en las posteriores décadas se referían a él, o por lo menos manifestaban una cierta inspiración en su obra.

En el marco de esta conmemoración, hay que mencionar a Alcide d'Orbigny, quien fue estimulado personalmente por Alexander von Humboldt y, en consecuencia, puede ser considerado uno de estos seguidores. En 1825, el joven Alcide D'Orbigny visita a Humboldt en París cuando empieza a preparar su propio viaje que le llevará entre 1826 y 1833 a las regiones de América del Sur.

Una prueba del significado que la obra y persona del sabio prusiano tuvieron para d'Orbigny es la dedicatoria que le hace en la obra El hombre americano (D'Orbigny, 1944):

"A quién podría yo haber pensado en dedicaros sino a vos; cuyo genio inspiró en cierto modo; a vos, a quien la Europa ha proclamado el modelo y el ejemplo de los viajeros filósofos!

Ojalá el mundo sabio pueda ver en el homenaje que yo rindo, la expresión del reconocimiento más sincero; ojalá halle en él la de los sentimientos que ya os consagré, como a uno de los más ilustres representantes de la ciencia contemporánea.

Demasiado feliz por no haber juzgado mi obra indigna de vos, Sr. Barón, cuán animado no me hallaré para continuar con los estudios a que consagré mi vida entera por una marca tan honrosa, que no cesa de haceros amigos, en una carrera en que ya no tenéis más rivales. 
Tengo la honra de ser con los más profundos sentimientos de respeto, Sr. Barón, vuestro más humilde y obediente criado, Alcide d'Orbigny."

También el texto necrológico de Font y Guitard (1862: 131) hace referencia a la importancia que tuvo Humboldt para otros científicos o viajeros, como vemos con las siguientes citas:

"De varios sabemos, y de algunos nos honramos con la amistad personal, que, sin la mediación de Humboldt, sin las sumas con que contribuyó a sustentarles, ni nunca llegarán a la posición que hoy ocupan, ni nunca hubieran podido llevar a término los trabajos con que se ilustraron, ni nunca hubieran conseguido la gloria que ellos se granjearon."

Más adelante (1862: 136) escribe:

"Para todos los viajes que se emprendían, solicitabase el patrocinio de Humboldt, se recorría a sus luces, quien trazaba el plan, conseguía subvenciones del erario público, y daba a los viajeros eficaces recomendaciones, con las cuales encontraba por donde quiera la protección más decidida por parte de los gobiernos. Una carta de Humboldt era, sobre todo en el nuevo continente, un pasaporte general, un crédito ilimitado. Así es que puede decirse que él formó principal parte de todos los viajes de exploración nuevamente intentados."

En este análisis no se puede dejar de hacer alusión a la repercusión que han tenido los tratados socio-político-económico que Humboldt realizó en el caso de México (1991) y de Cuba (1998). Los trabajos sistemáticos de estos países, con los mapas que ofrecieron una localización más precisa de los sitios y una descripción detallada, llegaron a ser los primeros estudios sociológicos de envergadura. Sus escritos no solamente contribuyeron de manera considerable a ampliar el conocimiento sobre ambos países, sino que inspiraron a muchos seguidores, quienes dieron a sus obras una orientación parecida. Como parte de su estela, se iniciaron una serie de ensayos sociológicos sobre diferentes países americanos. Ramón de la Sagra, para Cuba, y José María Luis Mora, para México, fueron algunos de estos seguidores.

$\mathrm{Al}$ introducir al lector a su famosa obra sobre México de 1836, México y sus revoluciones, Mora (1950, v. I: 4) hace de la siguiente manera referencia al Ensayo de Humboldt:

"De cuanto se ha escrito sobre la materia lo único digno de aprecio es el Ensayo político sobre el Reino de la Nueva España del barón de Humboldt. Esta obra clásica será siempre apreciada por el cuidado, diligencia y exactitud con que fueron acopiadas sus noticias. Son en ella de un interés permanente ciertos artículos por la naturaleza invariables, cualesquiera que sean los cambios políticos que el país haya tenido o pueda tener en lo sucesivo. En los otros si el Ensayo político no está exento de faltas, satisfizo por lo menos la expectación pública y dió a conocer a México como hasta entonces no lo había logrado ninguna obra."

Por su parte, la obra de Ramón de la Sagra, Historia económico-político y estadística de la isla de Cuba (1831), está próxima e inspirada en la de Humboldt, percibiéndose una cercanía a la corriente de modernidad iniciada por el prusiano, aunque La Sagra no lo expresara tan claramente como Mora. 
Esta recepción y mimetismo en algunos aspectos metodológicos de las obras de Humboldt demuestra otra de las contribuciones que realizó a la ciencia de aquel tiempo: pueden tener fallos o ser obsoletos por el contexto que ha cambiado, pero su valor está en su significado como modelo para un tratado sociológico, en el enfoque de su trabajo y, finalmente, en su función como inspiración científica.

Humboldt fue para las ciencias un vínculo entre la Ilustración y el Romanticismo. Esta unión entre el empirismo ilustrado y el idealismo romántico tanto en su metodología, su ideología, como en su planteamiento científico fue otro aporte suyo. De esta manera, en una época determinada por cambios, crisis, continuidades y rupturas, Humboldt ayudó a que el pensamiento evolucionara con una nueva orientación. En su obra se absorben distintos contextos culturales y sociales; el resultado es un modo particular de concebir el pensamiento científico y una forma distinta de aplicarlo a la lógica de su metodología (Monreal Sanz \& Álvarez Falcón, 2001, v. I: 349).

Los instrumentos científicos llamaron su interés durante toda su vida, no solamente se preocupó por tener lo más nuevo, y de aprender a utilizar con maestría estos instrumentos, sino que también se ocupó él mismo de su diseño. Ayudado por los instrumentos de medición más modernos, el naturalista alemán fue capaz de reunir una inmensa cantidad de datos a través de ellos (Sánchez Flores \& Seeberger,1997). La medición exacta de la realidad americana con estos instrumentos fue, sin duda, una de las aportaciones más significativas en el conocimiento científico del Nuevo Mundo.

Humboldt aunó todo el conocimiento europeo que se tenía sobre el continente americano, en las distintas disciplinas científicas, actualizó este saber y aportó a las ciencias europeas sus nuevos datos:

“(...) el resultado múltiple del viaje americano de Humboldt es amplio y no se puede reducir a ser el segundo descubrimiento de América. El viaje tiene consecuencias de primer orden para toda la ciencia: afina el método científico y amplía nuestra concepción del mundo." (Labastida, 1999: 50)

Finalmente, nos detendremos en la visión histórica que se ha tenido y transmitido de Humboldt en las antiguas fuentes, a través de la ya mencionada necrología, con el fin de evaluar qué relevancia científica se le confirió, qué aspectos de su obra se destacaron y que valor se dio a sus actividades. En este texto de Juan Font y Guitart hay que tener en cuenta que se trata de una necrología, lo que influye tanto en el estilo como en el contenido de lo escrito. Por lo tanto hay que contar con una visión extremadamente positiva y algo exagerada del personaje descrito. Sin embargo, hemos considerado útil incluirlo aquí puesto que una necrología muestra al final de una vida a qué se le atribuye el mérito y el significado de una persona.

En este artículo, que ya fue redactado en mayo del 1859 — es decir justo después de su muerte- el autor le llama "creador y cabeza de las ciencias populares" (Font y Guitard, 1862: 121), y haciendo referencia a su concepto científico, añade:

"Y no tan solo fué celoso y constante promovedor de todas las ramas de las ciencias, antes ya beneficiadas por otros ingenios, sino que algunas de ellas son enteramente creación suya propia; pues ni antes de él existieron la meteorología, la geografía vegetal, la climatología, la hidrografía, ni menos había nadie tentado la gigante empresa de describir en ordenado conjunto todos los fenómenos que se pasan en nuestro planeta y 
en su atmósfera: pues él es también el inmortal creador de la geografía física." (Font y Guitard, 1862: 130)

Claramente se muestra la idealización de Humboldt y de su importancia para las ciencias con frases del estilo siguiente:

"Ni en la antigüedad, ni los siglos modernos presentan el ejemplo de un barón tan completo, de un ánimo más levantado, de un espíritu más poderoso. Ha asistido, por decirlo así, al nacimiento de todas las ciencias naturales, y con el amor entrañable de un padre les prodigó sus fuerzas y sus desvelos; como ninguno, contribuyó a su desenvolvimiento, y llevolas enlazadas a su apogeo (...); en él se encarna, personifica y sublima todo el saber de su época; él corona la ciencia del siglo XIX." (Font y Guitard, 1862: 137)

En este artículo se destaca por un lado su espíritu emprendedor que le ha posibilitado llevar a cabo grandes empresas, mencionando su voluntad enérgica, su laboriosidad inconcebible, su trabajo perseverante, su actividad en todos los campos de la ciencia, así como la universalidad de su talento. Por otro lado, se hace especial énfasis en la aportación de su obra Cosmos a la ciencia:

“(...) el cuadro entero de la naturaleza fundado sobre la sólida base de la observación de los hechos. Esto era la parte más importante de su obra (...)”. (Font y Guitard, 1862: 131)

\section{REFLEXIÓN FINAL}

Haciendo este análisis de las contribuciones de Alexander von Humboldt a las ciencias, hay que ser conscientes que sus significados y valoraciones no fueron estáticas, sino que crecieron y se modificaron a lo largo de los años, en la medida en que la ciencia avanzaba y que las visiones del mundo variaban. Así se puede decir que a finales del siglo XVIII Humboldt aportó relevantes estudios en medicina y botánica. Durante su expedición americana y la posterior elaboración de sus memorias y diarios, el sabio alemán dio a conocer a la colectividad académica y al público en general muchas colecciones, datos, descripciones, y dibujos científicos. Finalmente, con el Cosmos como trabajo culminante de su vida, revelando conexiones entre los distintos aspectos del mundo, proporcionó una nueva visión de las ciencias. Los resultados de sus investigaciones fueron introducidos en el discurso científico actual de cada época, como refleja su resonancia en las publicaciones, así como en la prensa científica contemporánea. De esta manera, se sentaron las bases para una utilización de Humboldt como referencia científica, no solamente relacionado con sus propias investigaciones, sino como autoridad en general también en otros temas, lo que no excluye que hubiera siempre, como hubo ya a finales del siglo XVIII, también una fuerte crítica hacia la ciencia humboldtiana (Schipperges, 1990: 59-63).

En este contexto también es interesante las diferencias de utilización de los conocimientos y datos de Humboldt en distintos lugares y medios académicos. Mientras en Europa sus estudios sobre el Nuevo Mundo contribuyeron a que se modificara la visión de América, esto mismo hace que en América se le utilizara y utilice como mito, precisamente por la revaloración que realizó del continente (Lucena Giraldo, 1992: 
74). Hay que mencionar cómo muchos de sus comentarios referentes al colonialismo fueron utilizados por los criollos para fortalecer la reivindicación independentista (PuigSamper, 2000).

En este contexto hay que hacer una breve alusión a la propia visión que Humboldt tenía del desarrollo de las ciencias. El creyó firmemente en el progreso del siglo XIX, en que la humanidad se encontraba en un camino positivo imposible de detener. Por lo tanto, partió siempre de la idea de que sus investigaciones iban a ayudar en este proceso e iban a ser utilizadas siempre en un marco positivo y constructivo. En pos de ello, facilitó rápidamente sus conocimientos y los datos sin ser consciente —como pasó por ejemplo en el caso de México- que éstos también pueden ser utilizados para una mera explotación económica o para cuestiones militares en el área (Bernecker, 2001).

La visión histórica que nos han proporcionado estas fuentes de su recepción en el siglo XIX da una impresión de lo que se consideraron los aportes más importantes de Humboldt. En primer lugar está su concepto de la geografía física, con que Humboldt influyó mucho más en el futuro desarrollo de las ciencias que con sus investigaciones especializadas, además su obra sintetizadora el Cosmos, así como la popularización de las ciencias.

Humboldt tenía como punto de partida la comprensión y descripción de la realidad americana. Importante es, que este intento de representar el mundo a través de su perspectiva holística, surgió justo antes de que hacia la segunda mitad del siglo XIX comenzase la institucionalización de las ciencias como disciplinas académicas y con ello la especialización —y la separación- de estas disciplinas.

Como hemos visto, esta fragmentación de su pensamiento en disciplinas aisladas, como es característico para el mundo académico conservador a finales del siglo XIX, contribuyó de manera considerable a un cierto olvido de Humboldt. Hoy, en la época de la globalización, se redescubre la importancia de Humboldt para la creación de redes en el mundo científico (Arias Greiff, 2001: 176).

A pesar de la afirmación anterior hay que relativizar algunas de sus supuestas contribuciones ya que en muchos casos fue más un sintetizador de descubrimientos o de ideas preexistentes. Su imagen de América puede decirse que es original, aunque se basa en multitud de informes, documentos, etc. elaborados con anterioridad en territorio americano.

La pregunta de la validez actual de los resultados de las mediciones, observaciones así como de las conclusiones de la actividad científica de Humboldt no es un factor clave en la valoración de sus aportes hoy día. Como sabemos, con instrumentos más modernos y precisos se ha podido medir más exactamente la apariencia física de la realidad americana. Relacionado con sus estudios de tipo social o sociológico — si se quiere llamar así- es más evidente aún que su valor está justamente en esbozar una imagen de la sociedad de entonces. Las cifras de la composición de la población, su contexto social y político, la legislación vigente, las condiciones de vida de aquel tiempo habrán cambiado sustancialmente. Justamente en esas obras está una de las aportaciones más valiosas sobre todo para las ciencias sociales, ya que transmite una imagen de las sociedades coloniales americanas poco antes de la independencia. 
Concluyendo, se puede decir que aunque las hipótesis de Humboldt en algunos campos han tenido que ser modificadas o ampliadas, su obra contiene ideas que todavía son válidas. Como dice el geógrafo Horacio Capel (2000: 84):

"Todavía hoy nos produce un fuerte impacto intelectual su idea del estudio de la ciencia como 'un viaje a un país lejano', su convicción de que la investigación científica es un proceso que nunca se completará, la valoración de la investigación pura, que puede tener el germen de grandes descubrimientos aplicados, su énfasis en la conveniencia de desarrollar todos los caminos de la ciencia, su actitud optimista sobre la necesidad de investigar y conocer las leyes del universo para apropiarse de las fuerzas naturales y poder actuar sobre ellas."

\section{Referencias citadas}

ARIAS GREIFF, J., 2001 - Humboldt Begegnungen mit der Wissenschaft im spanischen Amerika: Transfer in zwei Richtungen. In: Alexander von Humboldt - Aufbruch in die Moderne (Ette, Ottmar, Ute Hermanns, Bernd M. Scherer \& Christian Suckow edits.): 169-178; Berlin: Akademie-Verlag.

BECK, H., 1978 - Alexander von Humboldt: Kosmos für die Gegenwart, 453p.; Stuttgart: Brockhaus.

BERNECKER, W. L., 2001 - Der Mythos vom mexikanischen Reichtum. Alexander von Humboldts Rolle vom Analysten zum Propagandisten. In: Ansichten Amerikas. Neuere Studien zu Alexander von Humboldt (Ottmar Ette \& Walther L. Bernecker edits.): 79103; Frankfurt a. M.: Vervuert Verlag. Lateinamerika-Studien, tomo 43.

BRUHNS, K. (ed.), 1969 - Alexander von Humboldt: Eine wissenschaftliche Biographie, 1346p.; Osnabrück: Otto Zell Verlag. 3 tomos (reedición del año 1872).

CAPEL, H., 2000 - De la armonía de la naturaleza a la física del globo. Las interrelaciones de la naturaleza terrestre durante el siglo XVIII. Quipu, enero-abril: 81-104.

DAUM, W., 1998 - Wissenschaftspopularisierung im 19. Jahrhundert: Bürgerliche Kultur, naturwissenschaftliche Bildung und die deutsche Öffentlichkeit, 1848-1914, 598p.; München: R. Oldenburg Verlag.

DETTELBACH, M., 2001 - Alexander von Humboldt zwischen Aufklärung und Romantik. In: Alexander von Humboldt. Aufbruch in die Moderne (Ette, Ottmar, Ute Hermanns, Bernd M. Scherer y Christian Suckow edits.): 137-149; Berlin: Akademie-Verlag.

D'ORBIGNY, A., 1944 - El hombre americano considerado en sus aspectos fisiológicos y morales, 423p.; Buenos Aires: Futuro (reedición del año 1839).

ETTE, O., 2000 - La puesta en escena de la mesa de trabajo en Raynal y Humboldt. In: La huella de Humboldt (Zea, L. edit): 31-67; México: Instituto Panamericano de Geografía e Historia. Latinoamérica Fin de Milenio 14.

FONT Y GUITARD, J., 1862 - Alejandro de Humboldt. La Abeja, 1: 121-138.

GERBI, A., 1982 - La disputa del Nuevo Mundo: Historia de una polémica 1750 - 1900, 884p.; México: Fondo de Cultura Económica.

HUMBOLDT, A. de, 1803 - Experimentos acerca del galvanismo y en general sobre la irritación de las fibras musculares y nerviosas, 537p.; Madrid: Imprenta de la Administración del Real Arbitrio de Beneficencia.

HUMBOLDT, A. von, 1860 - Briefe von Alexander von Humboldt und Varnhagen von Ense aus den Jahren 1827 bis 1858, 400p.; Leipzig: Brockhaus.

HUMBOLDT, A. von, 1874-1875 - Cosmos. Ensayo de una descripción física del mundo, 2168p.; Msxadrid: Imprenta de Gaspar y Roig. 4 tomos. 
HUMBOLDT, A. de, 1878 - Sitios de las Cordilleras y monumentos de los pueblos indígenas de América, 440p.; Madrid: Imprenta y librería de Gaspar.

HUMBOLDT, A. von, 1991 a - Viaje a las regiones equinocciales del Nuevo Continente, 2522p.; Caracas: Monte Avila Editores. 5 tomos.

HUMBOLDT, A. von,1991b - Ensayo político sobre el Reino de Nueva España, 698p.; México: Ed. Porrúa.

HUMBOLDT, A. de, 1992 - Cristóbal Colón y el descubrimiento de América, 393p.; Caracas: Monte Avila Editores.

HUMBOLDT, A. von, 1998 - Ensayo político sobre la isla de Cuba, 457p.; Aranjuez: Ediciones Doce Calles, Junta de Castilla-León. Estudio introductorio y edición de Miguel Ángel Puig-Samper, Consuelo Naranjo Orovio \& Armando García González. Theatrum Naturae. Colección de Historia Natural, Textos Clásicos.

JAHN, I. \& LANGE, F. G. (edits.), 1973 - Die Jugendbriefe Alexander von Humboldts 17871799, 838p.; Berlin: Akademie-Verlag.

JAHN, I., 2001 - Alexander von Humboldt's cosmical view on nature and his researchs shortly before and shortly after his departure from Spain. In: Estudios de Historia das Ciencias e das Técnicas. VII Congreso de la Sociedad Española de Historia de las Ciencias y de la Técnica: 31-39; Vol. I; Pontevedra.

LABASTIDA, J., 1999 - Una jornada de trabajo de Alexander von Humboldt: su método científico. Cuadernos Americanos, 76: 44-52.

LUCENA GIRALDO, M., 1992 - El espejo roto. Una polémica sobre la obra de Alejandro de Humboldt en la Venezuela del siglo XIX. Dynamis, 12: 73-8; Granada.

MINGUET, C., 1985 - Alejandro de Humboldt, historiador y geógrafo de la América española (1799-1804), 779p.; México: Universidad Nacional Autónoma de México. 2 tomos.

MONREAL SANZ, M. \& ÁLVAREZ FALCÓN, L., 2001 - Del racionalismo ilustrado a la sensibilidad romántica: La concepción singular del cambio de paradigma en la ciencia de Alexander von Humboldt. In: Estudios de Historia das Ciencias e das Técnicas. VII Congreso de la Sociedad Española de Historia de las Ciencias y de la Técnica: 349358; Vol. I; Pontevedra.

MORA, J. M. L., 1950 - México y sus revoluciones, 1317p.; México: Fondo de Cultura Económica. 3 tomos.

PUIG-SAMPER, M. A., 1999 - Humboldt, un prusiano en la Corte del Rey Carlos IV. Revista de Indias, Vol. LIX, 216: 329-355.

PUIG-SAMPER, M. A., 2000 - Alejandro de Humboldt y el mundo hispánico: La modernidad y la Independencia americana. Debate y perspectivas. Cuadernos de historia y ciencias sociales, 1; 251p.; Madrid: Fundación Histórica Tavera.

REBOK, S., 2001 - Alejandro de Humboldt y el modelo de la Historia Natural y Moral. Humboldt im Netz (revista de Internet: http://www.uni-potsdam.de/u/romanistik/humboldt/hin), 3; Berlín/Potsdam.

ROJAS-MIX, M., 1969 - Die Bedeutung Alexander von Humboldts für die künstlerische Darstellung Lateinamerikas. In: Alexander von Humboldt. Werk und Weltgeltung (Heinrich Pfeiffer, edit.): München; R. Piper \& Co. Verlag.

SAGRA, R. de la, 1831 - Historia económico-político y estadística de la isla de Cuba, 386p.; La Habana: Imprenta de las Viudas de Arazoza y Soler.

SÁNCHEZ FLORES, R. \& SEEBERGER, M., 1997 - Humboldt y sus instrumentos científicos. In: Alejandro de Humboldt en México: 57-65; México: Instituto Nacional de Antropología e Historia.Catálogo de la exposición.

SCHIPPERGES, H., 1990 - Humboldts Beitrag zur Medizin des 18. Jahrhunderts. In: Miscellanea Humboldtiana (Kurt- R. Biermann, edits): 37-68; Berlin: Akademie-Verlag. 